\title{
Postoperative striatal degeneration: a hitherto unrecognized impact of frontal disconnection surgery for drug-resistant epilepsy. Illustrative cases
}

\author{
Koichi Hagiwara, MD, PhD, ${ }^{1}$ Hideaki Tanaka, MD, PhD, ${ }^{2}$ Ayako Miyoshi, MD, ${ }^{1}$ Takashi Kamada, MD, PhD, ${ }^{1}$ Hiroshi Shigeto, MD, PhD, ${ }^{1,3}$ \\ Shinji Ohara, MD, PhD, ${ }^{1,2}$ and Naoki Akamatsu, MD, PhD ${ }^{1}$ \\ ${ }^{1}$ Epilepsy and Sleep Center, ${ }^{2}$ Department of Neurosurgery, Fukuoka Sanno Hospital, Fukuoka, Japan; and ${ }^{3}$ Division of Medical Technology, Department of Health Sciences, \\ Graduate School of Medical Sciences, Kyushu University, Fukuoka, Japan
}

\begin{abstract}
BACKGROUND Frontal disconnection surgery is a useful surgical option for patients with frontal epilepsy whose seizure onset zones are exceedingly large and thus are not amenable to conventional resective surgery. While it has the advantage of avoiding sequelae stemming from a large resection cavity, the impact of radical anatomofunctional disconnection of such a vast frontal region is not fully understood.

OBSERVATIONS The authors have identified secondary degeneration in the striatum ipsilateral to the frontal disconnection surgery in two adult patients who had otherwise favorable postoperative outcomes following the surgery. On serial postoperative magnetic resonance imaging, the striatum showed transient restricted diffusion in the caudate head and rostral putamen around several weeks postoperatively and subsequent atrophy in the caudate head. The affected striatal regions (i.e., the anterior portion of the striatum) were congruent with the known fronto-striatal connectivity corresponding to the disconnected frontal regions anterior to the primary and supplementary motor areas. Both patients achieved 1-year seizure freedom without apparent disability related to the surgery.
\end{abstract}

LESSONS The benign postoperative course despite the marked degenerative changes in the ipsilateral striatum supports the feasibility of the frontal disconnection surgery in otherwise inoperable patients with broad frontal epileptogenicity.

https://thejns.org/doi/abs/10.3171/CASE21644

KEYWORDS frontal disconnection surgery; frontal epilepsy; fronto-striatal connectivity; reduced diffusion; striatum; Wallerian degeneration

Frontal disconnection surgery, also called peri-insular anterior quadrantotomy, has been proposed as an effective surgical option for drug-resistant epilepsy with widespread frontal epileptogenicity. ${ }^{1,2}$ This technique involves functional disconnection of the frontal lobe harboring a vast epileptogenic zone while preserving the eloquent areas. Such disconnectivetype surgery allows avoiding cavity-related complications (e.g., hematoma, hydrocephalus) associated with a large brain resection. ${ }^{3}$

Literature on this procedure is still scarce. In fact, the disconnective technique involving the frontal lobe has been reported in recent years, ${ }^{1,2}$ and thus far, only a single case series has assessed the efficacy and the safety of this procedure. ${ }^{2}$ While it appears to be a viable surgical option, the impact of such extensive anatomofunctional disconnection is not fully understood.
Herein we report two patients who had successful outcomes after frontal disconnection surgery despite leaving behind secondary degenerative changes in the ipsilateral striatum.

\section{Illustrative Cases \\ Case 1}

A 37-year-old right-handed female nursery teacher experienced the onset of epilepsy at age 15. She did not have any prior medical conditions or family history of epilepsy. Her seizure semiology consisted of coordinated repetitive tapping movements with both her arms while maintaining her consciousness. She described her ictal motor behavior as "being unable to stop clapping." Her seizures were brief, lasting 10 to 15 seconds without impaired awareness

ABBREVIATIONS ${ }^{18} \mathrm{FDG}$-PET $=\left[{ }^{18} \mathrm{~F}\right]$-fluorodeoxyglucose positron emission tomography; $\mathrm{EEG}=$ electroencephalography; FLAIR = fluid-attenuated inversion recovery; $M R I=$ magnetic resonance imaging; SEEG = stereo-electroencephalography.

INCLUDE WHEN CITING Published February 28, 2022; DOI: 10.3171/CASE21644.

SUBMITTED November 11, 2021. ACCEPTED January 13, 2022.

(c) 2022 The authors, CC BY-NC-ND 4.0 (http://creativecommons.org/licenses/by-nc-nd/4.0/). 


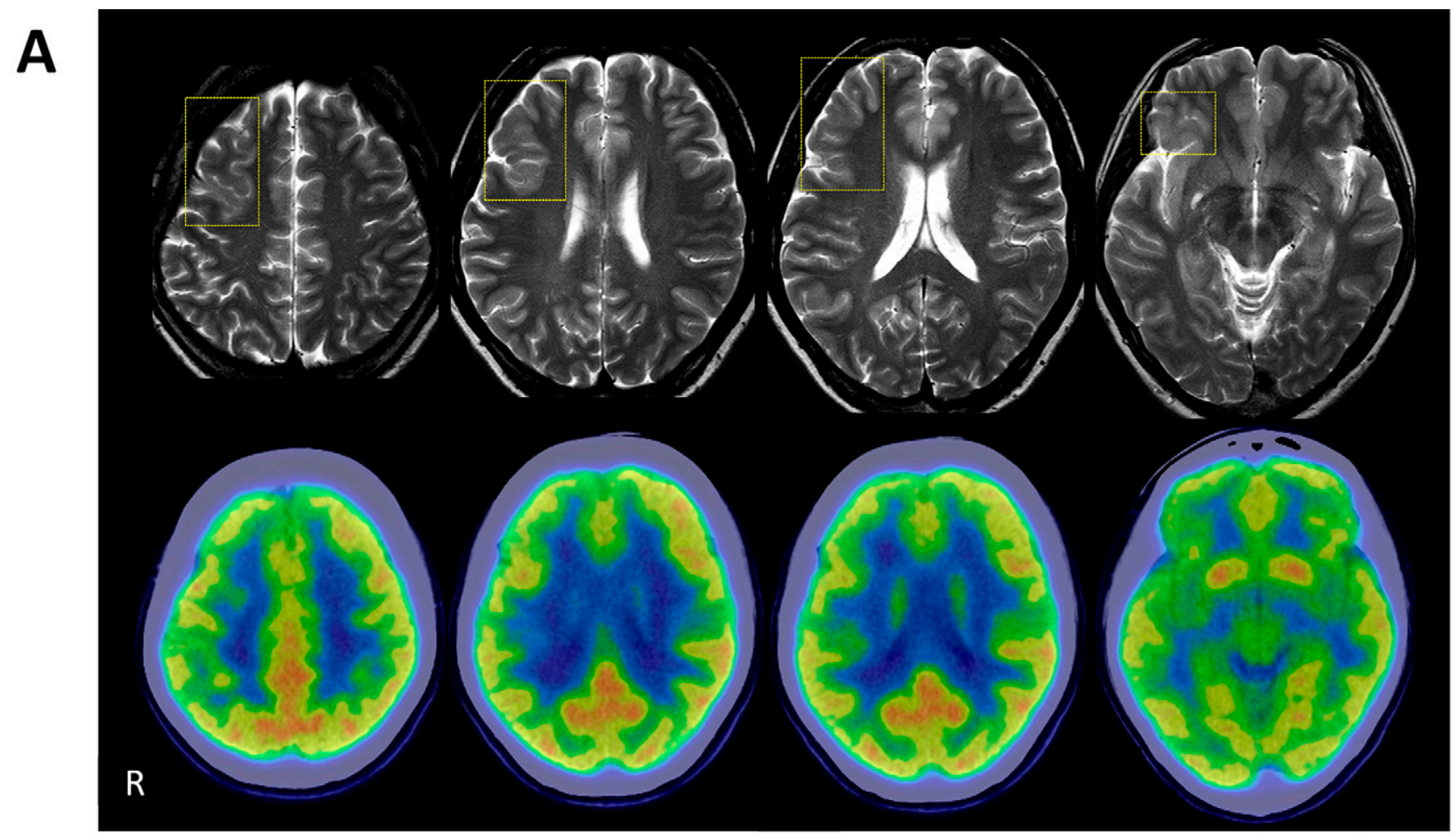

B
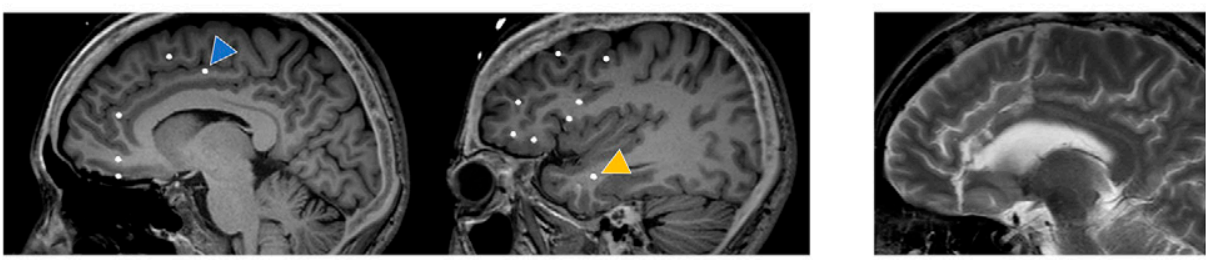

Medial Prefrontal

Midcingulate

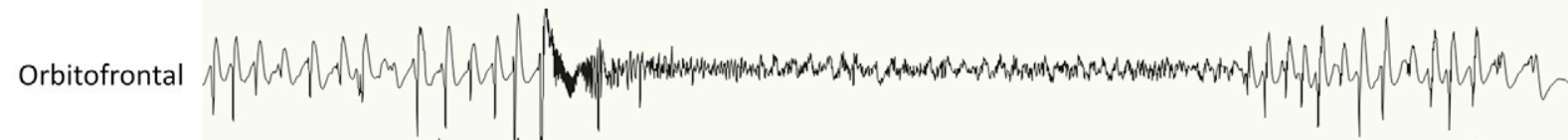

Lateral Prefrontal

$1 \mathrm{sec}$

Temporal $\left\{\begin{array}{l}\text { Mesial } \\ \text { Lateral }\end{array}\right.$

FIG. 1. Noninvasive and invasive investigation results for case 1. A: MRI showing a widespread dysplastic cortex in the right frontal lobe (upper, highlighted with a yellow rectangle) and 18FDG-PET showing a hypometabolism in the same region (lower). B: SEEG investigation. The intracortical electrode positions are indicated by white dots on the MRI. Ictal fast discharges were widely distributed in the frontal lobe, but the posterior frontal (midcingulate) and temporal regions were spared (indicated by blue and orange arrowheads, respectively). The frontal disconnection line was set at the rostral limit of the supplementary motor area, which was indicated by the midcingulate electrode (see upper right MRI for the medial disconnection line). We did not explore the primary motor area due to the absence of tonic-clonic contractions during her seizures. The lateral disconnection line was therefore set anterior to the precentral gyrus (not shown). 

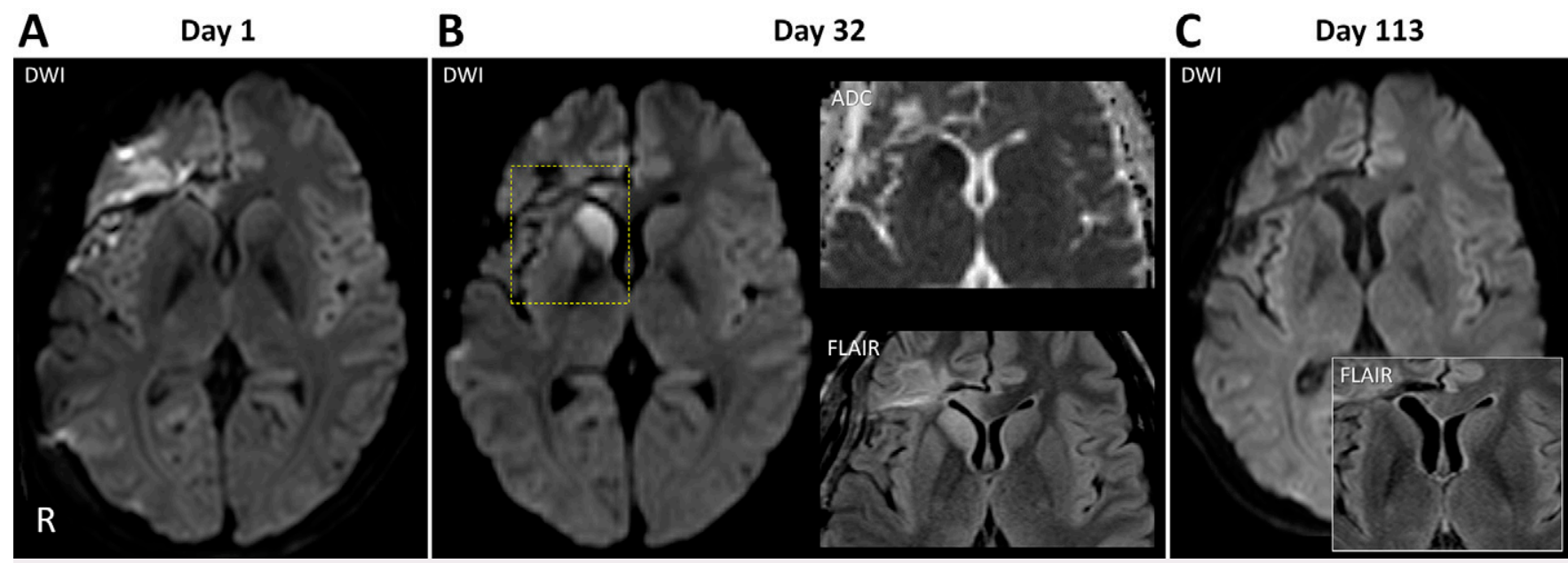

FIG. 2. Serial postoperative MRI for case 1. On the first postoperative day (A), no abnormal signal other than the periprocedural one (i.e., the disconnection line) was observed on the diffusion-weighted image (DWI). On the 32nd postoperative day (B), the DWI showed high signals in the caudate head, and also slightly in the rostral putamen (highlighted with a yellow rectangle in the left image). The apparent diffusion coefficient (ADC) map showed a low signal in the corresponding striatal region, thus exhibiting a restricted diffusion pattern (upper right). The FLAIR image showed a high signal in the same region (lower right). On the 113th postoperative day (C), the restricted diffusion had completely resolved, but the caudate head was found to be atrophied.

(i.e., focal aware automatism seizure), followed by abrupt resumption of normal motor control. Despite trials of multiple antiepileptics, her seizures occurred several times a day, both during the daytime and sleep, and thus seriously affected her professional life as a nursery teacher. Her interictal scalp electroencephalography (EEG) showed frequent Fp2-F8 spikes or spike bursts. Her seizures started with a large-amplitude initial spiking with a predominance in the right frontal region ( $F 8, F 4)$, followed by low-amplitude fast discharges in the same distribution. Cranial magnetic resonance imaging (MRI) showed dysplastic sulci and atrophy with mild T2-fluid-attenuated inversion recovery (FLAIR) hyperintensities in the right orbitofrontal cortex and the middle frontal gyrus (Fig. 1A). $\left[{ }^{18} \mathrm{~F}\right]$-fluorodeoxyglucose positron emission tomography $\left({ }^{18} \mathrm{FDG}-\mathrm{PET}\right)$ showed a hypometabolism involving the entire right frontal lobe (Fig. 1A). Stereo-electroencephalography (SEEG) revealed broadly distributed ictal fast discharges in the orbitofrontal region, medial as well as lateral prefrontal regions, and the premotor region (Fig. 1B). Due to the lack of a focal onset amenable to standard corticectomy or lobectomy, she underwent a right frontal disconnection surgery while sparing the primary and supplementary motor areas. A small corticectomy was performed in the posterodorsal portion of the middle frontal gyrus (i.e., the premotor region), but histological examination using this tissue specimen yielded no specific findings. She had no neurological deficits, and postoperative MRI 1 day after surgery confirmed the successful disconnection without perioperative ischemic/hemorrhagic complications (Fig. 2A). Abrupt seizure cessation after surgery was accompanied

TABLE 1. Pre- and postoperative neuropsychological performance

\begin{tabular}{|c|c|c|c|c|}
\hline \multirow[b]{2}{*}{ Test } & \multicolumn{2}{|c|}{ Case 1} & \multicolumn{2}{|c|}{ Case 2} \\
\hline & Preoperative & Postoperative & Preoperative & Postoperative \\
\hline WAIS-III, VIQ & 78 & 82 & 64 & 93 \\
\hline WAIS-III, PIQ & 74 & 80 & 71 & 94 \\
\hline WAIS-III, FSIQ & 74 & 76 & 64 & 93 \\
\hline WAIS-III, WMI & 76 & 79 & 69 & 79 \\
\hline WAIS-III, VCl & 64 & 82 & 64 & 97 \\
\hline WAIS-III, PSI & 78 & 89 & 75 & 84 \\
\hline WAIS-III, PRI & 83 & 79 & 83 & 108 \\
\hline WMS-R, Verbal memory & 81 & 83 & 68 & 75 \\
\hline WMS-R, Visual memory & 90 & 108 & 100 & 81 \\
\hline WMS-R, Total memory & 81 & 88 & 74 & 74 \\
\hline WMS-R, Delayed recall & 70 & 88 & 68 & 68 \\
\hline WMS-R, Attention & 94 & 94 & 87 & 79 \\
\hline
\end{tabular}

$\mathrm{FSIQ}=$ full scale intelligence quotient; $\mathrm{PIQ}=$ performance intelligence quotient; $\mathrm{PRI}=$ perceptual reasoning index; $\mathrm{PSI}=$ processing speed index; $\mathrm{VCl}=$ verbal comprehension index; VIQ = verbal intelligence quotient; WAIS-III = Wechsler Adult Intelligence Scale 3rd edition; WMS-R = Wechsler Memory Scale-Revised; WMI = working memory index. 
by a transient depressive state with anxiety (i.e., alternative psychosis) lasting approximately 3 months postoperatively. Follow-up MRI on the 32nd postoperative day revealed restricted diffusion with T2-FLAIR signal hyperintensity in the right caudate nucleus and, to a lesser degree, in the rostral putamen (Fig. 2B). Magnetic resonance angiography was normal. On the 113th postoperative day, these signal abnormalities resolved spontaneously, but the head of the caudate nucleus was atrophied
(Fig. 2C). No cognitive decline was observed postoperatively (Table 1). She remained seizure-free at 1 -year follow-up.

\section{Case 2}

A 46-year-old right-handed male ironworker experienced the onset of epilepsy at age 27 . He had no past or family history of neurological conditions other than his epilepsy. His seizures started with sudden,

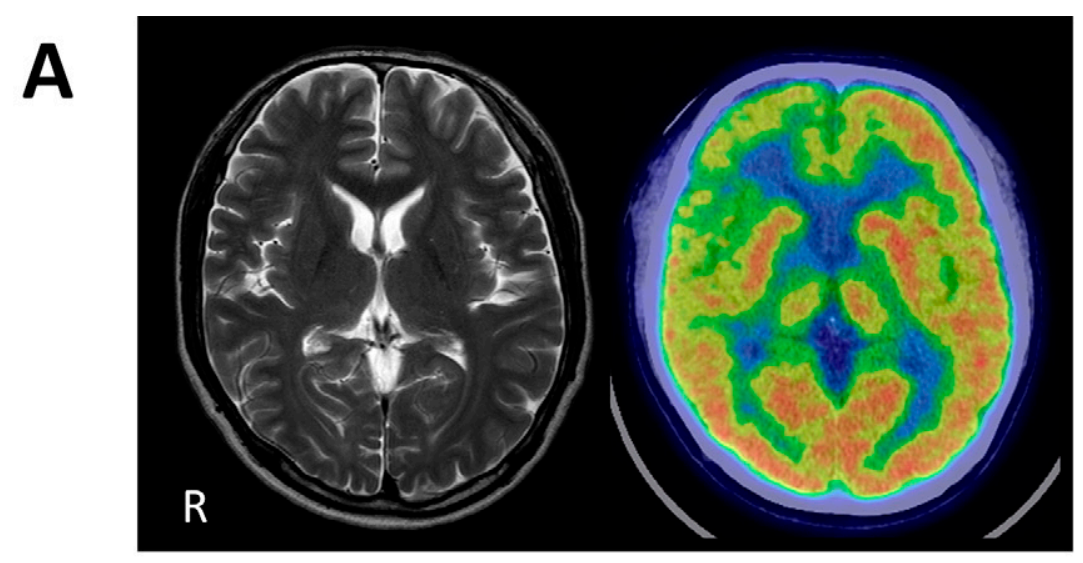

B
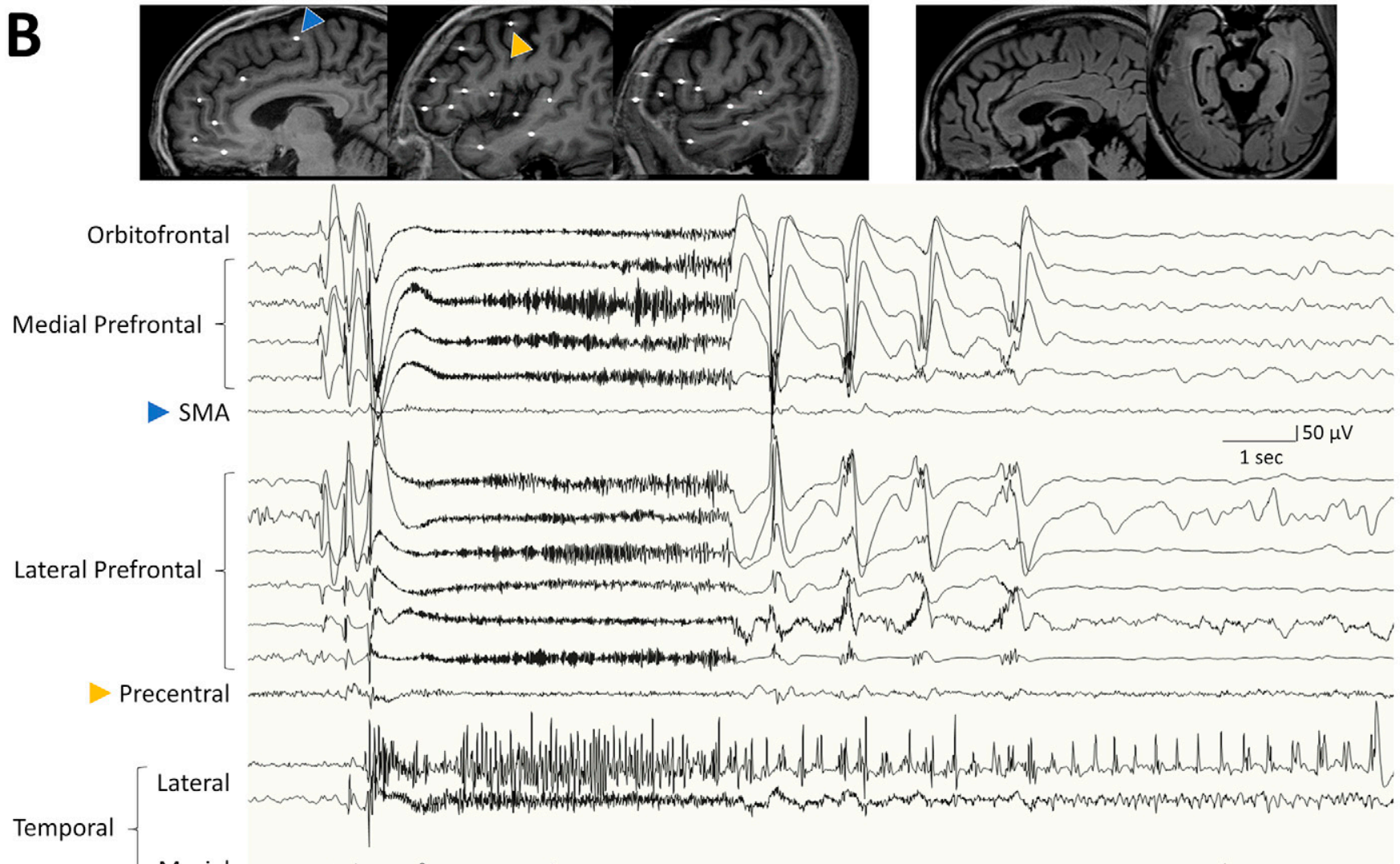

Mesial

FIG. 3. Noninvasive and invasive investigation results for case 2. A: MRI showed no abnormality (left), whereas 18FDG-PET disclosed a vast hypometabolism in the right hemisphere, mainly involving the fronto-temporal region (right). B: SEEG investigation. The intracortical electrode positions are indicated by white dots on the MRI. Ictal fast discharges involved the entire prefrontal and orbitofrontal regions, and they spread rapidly to the lateral temporal neocortex. Only the supplementary and primary motor (precentral) areas were spared from the ictal discharges (indicated by blue and orange arrowheads, respectively). The upper right MRI shows the medial disconnection line crossing the rostral supplementary motor area. The lateral disconnection line was set anterior to the precentral gyrus (not shown). A corticectomy (subpial aspiration) was performed in the lateral temporal region (see also the upper right MRI). 


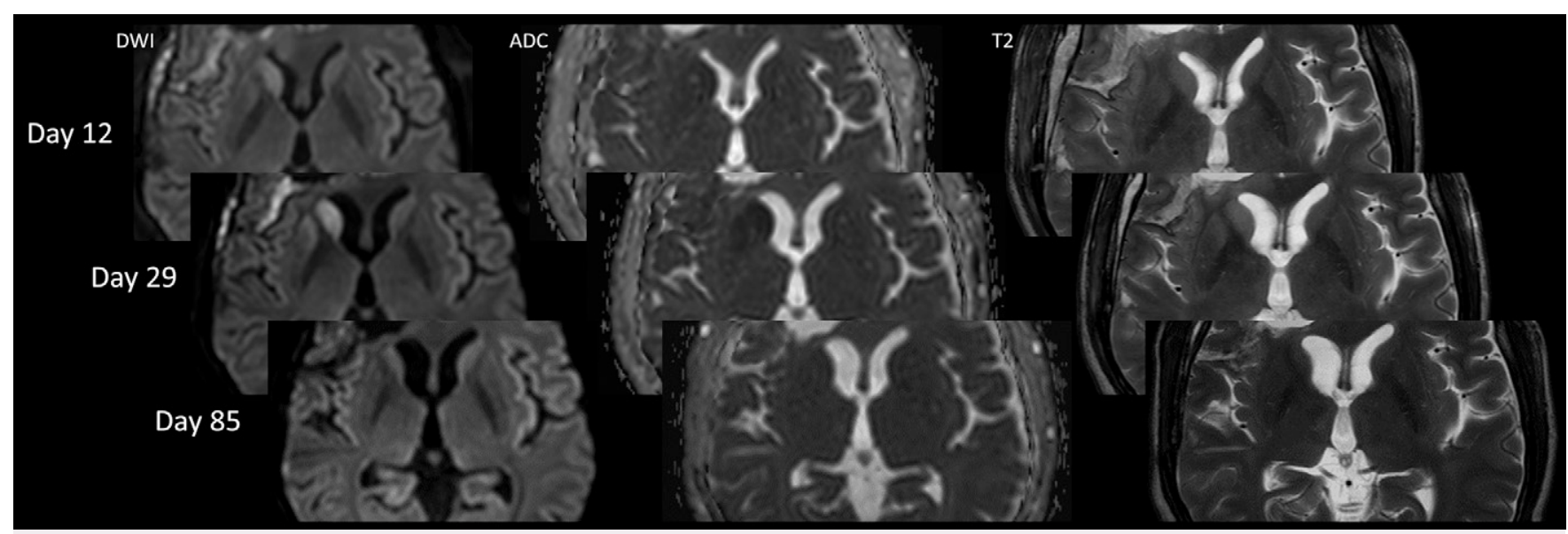

FIG. 4. Serial postoperative MRI for case 2. The restricted diffusion pattern and T2 hyperintensity were emerging in the caudate head and the rostral putamen on the 12th postoperative day (upper). The signal abnormalities became intense on the 29th postoperative day (middle). These signal abnormalities had completely resolved, but there was atrophy of the caudate head on the postoperative day 85 (lower). DWI = diffusion-weighted image; $A D C=$ apparent diffusion coefficient.

repetitive stroking of the head with his right hand while lacking his awareness (i.e., focal impaired awareness automatism seizure), followed by verbalization of nonsense words and purposeless coordinated waving movements of his upper limbs. Each seizure lasted less than 20 seconds and ended with an immediate resumption of normal voluntary activity. They were resistant to antiepileptics and were witnessed almost daily by his coworkers. Interictal scalp EEG showed F4-F8 spikes, often with wide distribution in the right hemisphere. Ictal EEG showed a similar spike and succeeding low-amplitude fast discharges in the right frontotemporal distribution. Brain MRI was unrevealing, whereas ${ }^{18} \mathrm{FDG}$-PET disclosed a vast right frontal as well as lateral temporal hypometabolism (Fig. 3A). Consistent with these noninvasive results, SEEG showed widespread ictal fast discharges over the right fronto-temporal region: only the primary and supplementary motor areas were spared from the seizure discharges in the frontal lobe, and early propagation to the superior and middle temporal gyri was observed (Fig. 3B). He underwent a frontal disconnection surgery combined with the subpial aspiration of the two temporal gyri. The frontal disconnection line was set anterior to the primary and supplementary motor areas. Histopathologic examination was not available since we did not perform en bloc resection. Postoperatively he experienced no neurological or neuropsychological complications. Because of the previous experience with the patient in case 1, we performed serial follow-up MRI. On the 12th postoperative day, there was slightly restricted diffusion in the right caudate head (Fig. 4, upper). There was no indication of vasospasm. On the 29th postoperative day, the signal abnormalities became even more intense and also involved the rostral putamen (Fig. 4, middle). No signal abnormalities were observed on the 85th postoperative day, but there was atrophy of the caudate head (Fig. 4, lower). He achieved 1-year seizure freedom, and an overall improvement in his cognitive performance was also observed (Table 1).

\section{Discussion}

\section{Observations}

SEEG demonstrated broad frontal epileptogenicity anterior to the primary and supplementary motor areas in both patients. The absence of tonic/clonic contractions during their seizures reflected the lack of propagation in these posterior frontal regions. Therefore, the frontal disconnection was a suitable measure to achieve seizure freedom. Their postoperative outcomes regarding both seizures and cognitive functioning were favorable, despite the gradual development of striatal abnormalities ipsilateral to the disconnection surgery.

The frontal disconnection surgery is a practical surgical option in cases where intracranial EEG investigation is confronted with broadly distributed epileptogenic foci involving the anterior noneloquent frontal region. ${ }^{2}$ However, such widespread disconnection is likely to have a nonnegligible impact on the remaining brain networks. Obviously, the rich fronto-striatal connections ${ }^{4-7}$ are widely disrupted by this procedure. In our patients, the serial postoperative follow-up MRI showed transient restricted diffusion with subsequent atrophy in the ipsilateral striatum. Perioperative ischemia in the anterior perforator territory ${ }^{8}$ was unlikely because the restricted diffusion developed gradually over a month and was not accompanied by clinical signs of acute ischemia, angiographic evidence of vasospasm, or chronic signal changes suggesting the vascular etiology (see the next paragraph for further details). Such late occurrence of restricted diffusion in the striatum suggests secondary neurodegeneration (i.e., Wallerian degeneration) due to the widespread frontostriatal disconnection and has been reported to occur in a proportion of patients with resective-type frontal surgery. ${ }^{9}$ The restricted diffusion was transient, reaching their peaks around 2 to 3 weeks after the surgery ${ }^{9}$ as in our patients. However, similar findings have not yet been reported in patients who underwent frontal disconnection surgery. ${ }^{2}$ The lack of specific neurological symptoms is probably the reason to have missed the appropriate time window to observe the transient striatal signal abnormalities in the previous cases of frontal disconnection.

One may raise the possibility of delayed-onset vasospasm involving the Heubner's and/or lenticulostriate arteries as the underlying etiology. However, magnetic resonance angiography did not suggest this possibility, and the gradual intensification of signal changes over a month is unlikely to occur in vasospasm. In addition, there were no signs of acute ischemia involving the caudate and adjacent regions such as contralateral hemiparesis, dysarthria, 
behavioral changes (e.g., abulia, slowness of thought, or restlessness/agitation), or choreoathetosis. ${ }^{10}$ The postoperative depression with anxiety in the patient in case 1 was dissimilar to these behavioral changes and can be explained by the alternative psychosis. Furthermore, ischemic lesions show more or less edema unless the image was taken in the hyperacute period, and they also develop residual T2-FLAIR hyperintensity or a cavitary change in the chronic period. Our patients developed none of these secondary signal changes.

The reduced diffusion was limited to the caudate head and the rostral putamen. The anterior striatum is densely connected with the orbitofrontal, prefrontal, and anterior cingulate cortices but less with the premotor and motor cortices, and vice versa for the posterior striatum. ${ }^{4-7}$ As the supplementary and primary motor areas were the only frontal regions preserved in our patients, the posterior striatum was spared from the Wallerian degeneration.

We acknowledge that there was a slight technical modification from the originally proposed disconnection procedure: ${ }^{1,2}$ the anterior corpus callosotomy was not performed because it was considered nonessential in our patients. However, this did not affect their seizure outcomes, nor appeared to be related to the occurrence of striatal degeneration.

\section{Lessons}

In conclusion, our cases showed that the ipsilateral striatum can undergo secondary neurodegeneration following the frontal disconnection surgery. The striatal signal abnormalizes should not be confused with perioperative ischemic stroke. ${ }^{9}$ They may pass unnoticed due to the lack of obvious neurological symptoms likely because the procedure is performed in patients with a vast frontal lesion or hypometabolism. The benign postoperative course despite the striatal degeneration supports the use of this surgical procedure in otherwise inoperable patients with broad frontal epileptogenicity.

\section{Acknowledgments}

We would like to thank the EEG technicians at Epilepsy and Sleep Center, Fukuoka Sanno Hospital, Fukuoka, Japan for their help in acquiring the electrophysiological data of the patients.

\section{References}

1. Cossu G, Lebon S, Seeck M, et al. Periinsular anterior quadrantotomy: technical note. J Neurosurg Pediatr. 2018;21(2):124-132.
2. Kamalboor $\mathrm{H}$, Alhindi $\mathrm{H}$, Alotaibi F, Althubaiti I, Alkhateeb M. Frontal disconnection surgery for drug-resistant epilepsy: outcome in a series of 16 patients. Epilepsia Open. 2020;5(3):475-486.

3. Daniel RT, Meagher-Villemure K, Farmer JP, Andermann F, Villemure JG. Posterior quadrantic epilepsy surgery: technical variants, surgical anatomy, and case series. Epilepsia. 2007;48(8): 1429-1437.

4. Lehéricy S, Ducros M, Van de Moortele PF, et al. Diffusion tensor fiber tracking shows distinct corticostriatal circuits in humans. Ann Neurol. 2004:55(4):522-529.

5. Morris LS, Kundu P, Dowell N, et al. Fronto-striatal organization: defining functional and microstructural substrates of behavioural flexibility. Cortex. 2016;74:118-133.

6. Haber SN. Corticostriatal circuitry. Dialogues Clin Neurosci. 2016;18(1):7-21.

7. Graff-Radford J, Williams L, Jones DT, Benarroch EE. Caudate nucleus as a component of networks controlling behavior. Neurology. 2017;89(21):2192-2197.

8. Khan NR, Moore K, Basma J, et al. Ischemic stroke following elective craniotomy in children. J Neurosurg Pediatr. 2018;23(3): 355-362.

9. Kamiya K, Sato N, Nakata Y, et al. Postoperative transient reduced diffusion in the ipsilateral striatum and thalamus. AJNR Am J Neuroradiol. 2013;34(3):524-532.

10. Caplan LR, Wechsler L. Large artery occlusive disease of the anterior circulation. In: Caplan L, ed. Caplan's Stroke: A Clinical Approach. Cambridge University Press; 2016:217-251.

\section{Disclosures}

The authors report no conflict of interest concerning the materials or methods used in this study or the findings specified in this paper.

\section{Author Contributions}

Conception and design: Hagiwara, Akamatsu. Acquisition of data: Hagiwara, Tanaka, Miyoshi, Shigeto, Ohara, Akamatsu. Analysis and interpretation of data: Hagiwara, Akamatsu. Drafting the article:

Hagiwara. Critically revising the article: Hagiwara, Shigeto. Reviewed submitted version of manuscript: Hagiwara, Shigeto, Akamatsu. Approved the final version of the manuscript on behalf of all authors: Hagiwara. Administrative/technical/material support: Tanaka, Kamada. Study supervision: Hagiwara, Akamatsu.

\section{Correspondence}

Koichi Hagiwara: Epilepsy and Sleep Center, Fukuoka Sanno Hospital, Fukuoka, Japan. hagiwara-kyu@umin.ac.jp. 